\title{
Trust without Control
}

\section{Dear Reader,}

Do you know what strawberries, dollhouses and cat food have in common? Well, they are among the products that unsuspecting people had delivered to their doors unexpectedly, because they had not ordered them. The culprit was the Amazon smart speaker which springs into action when someone says "Alexa." And that's how amusing mistakes like these do sometimes happen. What is less funny is when Alexa records people in their bedroom, and then sends the recordings to third parties, which has also happened. These are cases of undesirable interference and a serious lack of data security in an assistance system or, more accurately, in a humanmachine interface, because that is precisely what Alexa is.

Human-machine interfaces in cars, which offer occupants an increasing range of options for interacting with the car's various functions, need to be more reliable than a system that we use sitting at home on our sofa. In the car, we are in the highly sensitive area of real and perceived safety on the roads. The rapidly growing variety of technical possibilities, for example holographic displays that float freely inside the car and can be operated using simple hand gestures, presents developers with new challenges. In a future defined by augmented reality, how can they ensure that it is almost impossible to give an incorrect command?

During the development of HMI systems, car manufacturers would be well advised to put a lot of effort into ensuring that the systems function safely and are accepted by customers, and not only for the reasons given above. Car users will be transformed from people who take actions into objects which have to be monitored by the cameras and sensors that form part of the systems in their vehicles. On all levels of automated driving right up to SAE level 5, it will be necessary for the technical systems to know about the status of the driver. This is the only way in which responsibility for driving can safely be transferred from the machine to the person and vice versa, as IAV explains in its article on fallback systems on page 26.

The Latin verb "assistere" means "to stand nearby” or "to support.” An assistance system, particularly in a car, must therefore never become a Big Brother that can make decisions on behalf of the people entrusted to its care. Nevertheless in many respects we will eventually have to stop using the saying "Trust is good - control is better," because we will ultimately be handing full control over to the systems. And, as far as the purely driving-related functions are concerned, that is definitely something to look forward to.

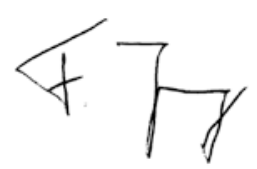

Frank Jung

Editor

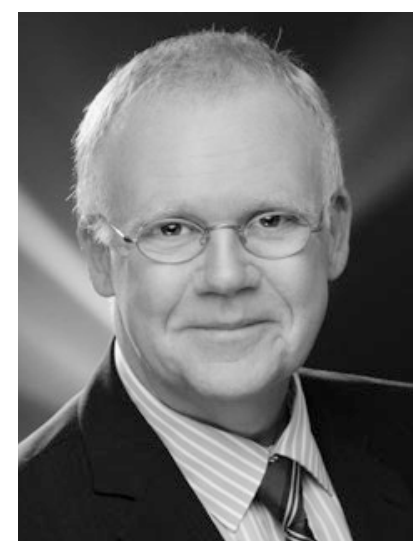

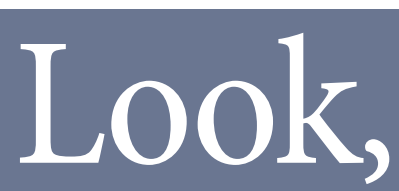

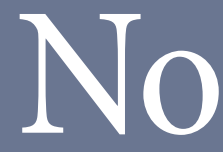

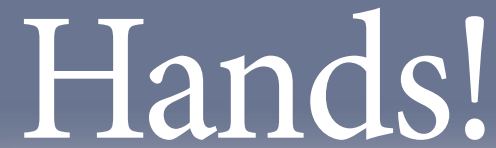

Just a matter of the right methods.

Driver assistance systems are changing the very nature of cars. Connected and increasingly open, these security-critical assistants can shake the very foundations of conventional wisdom. We strike the right balance and put it all on a solid footing by combining systems engineering, safety, security and smart testing methods. It all comes together in one unified function-driven concept. And our solutions are scalable and reusable to give you even more avenues to explore.

ITK Engineering GmbH - Take the open road to driving freedom.

www.itk-engineering.com

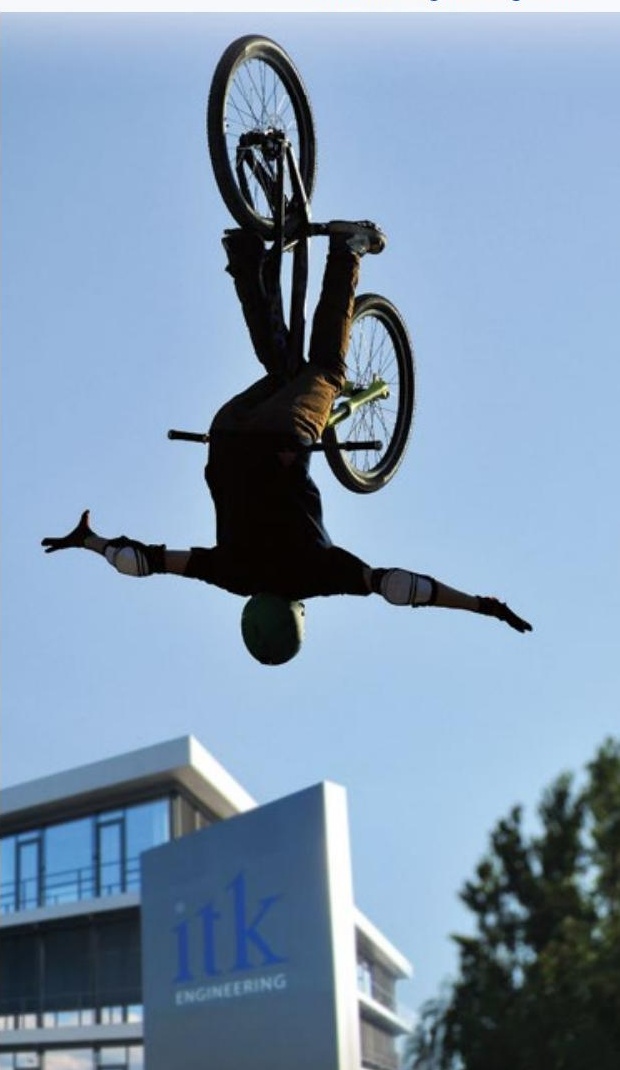

